\title{
Realidade virtual no tratamento do transtorno de pânico
}

\author{
Virtual reality in treatment of panic disorder
}

Marcele Regine de Carvalho', Rafael C. Freire', Antonio Egidio Nardi²

\section{RESUMO}

O uso de realidade virtual (RV) é útil no tratamento de exposição em pacientes com transtorno de pânico (TP). Em ambientes virtuais, os pacientes experimentam alterações fisiológicas, psíquicas e somáticas similares aos sintomas em situações da vida real, facilitando o processo de habituação. Objetivo: Divulgar o panorama atual do uso de RV no tratamento do TP. Método: A literatura sobre o tratamento do TP a partir da RV foi revisada com metodologia sistemática na base de dados PubMed, por meio das palavras-chave: virtual reality e panic disorder. Resultados: $\mathrm{O}$ tratamento do TP com RV está em geral associado a

\section{Palavras-chave}

Transtorno de pânico, terapia cognitivocomportamental, realidade virtual, exposição.

\section{Key-words}

Panic disorder, cognitive-behavior therapy, virtual reality, exposure therapy. bons resultados e à boa aceitabilidade por parte dos pacientes. São poucos os estudos que mensuram respostas fisiológicas durante a exposição, apesar da importância destes dados para avaliação da eficácia do tratamento. Conclusões: Apesar da utilidade do tratamento do TP por RV ser verificada nos estudos, constata-se necessidade de estudos controlados e protocolos de tratamento padronizados.

\section{ABSTRACT}

Virtual reality (VR) is a valuable tool in exposure treatment of panic disorder (PD). In virtual environments the patients experience similar physiological, psychic and somatic changes as in real life situations facilitating the habituation process. Objective: We intend to divulge the current panorama of VR in treatment of PD. Method: The literature on VR treatment for PD was revised with a systematic methodology in the PubMed database, using the keywords: virtual reality and panic disorder. Results: Virtual exposure treatment of PD usually shows good results and a great patient acceptability. Only a few studies measure physiological responses during the exposure despite the importance of these data for evaluation of the treatment effectiveness. Conclusions: Although the VR treatment for PD usefulness is clear in the trials, a lack of controlled studies and standardized treatment protocols are verified.

Laboratório de Pânico e Respiração; Instituto de Psiquiatria; Universidade Federal do Rio de Janeiro (UFRJ).

1 Programa de Pós-Graduação em Psiquiatria e Saúde Mental do Instituto de Psiquiatria da Universidade Federal do Rio de Janeiro (UFRJ). 2 Instituto de Psiquiatria da Faculdade de Medicina da UFRJ. 


\section{INTRODUÇÃO}

A introdução de novas tecnologias no campo da saúde mental pode ser uma forma de potencializar a eficácia ou expandir possibilidades de diagnóstico e intervenção de tratamentos tradicionais. No campo da psicoterapia, uma grande inovação que vem atualmente sendo aperfeiçoada em virtude do avanço tecnológico é o uso de realidade virtual. Wiederhold' ilustra este cenário com o aumento do número de exposições e publicações sobre realidade virtual na psiquiatria e na psicologia na década de 1990. Em 1993 nenhuma publicação foi encontrada e apenas duas apresentações profissionais foram feitas sobre o tema. Já em 1999, foram realizadas 77 apresentações e 53 artigos foram publicados. O ápice deste período ocorreu em 1998, com 61 publicações. Atualmente, apenas na base de dados PubMed, uma busca simples pela palavra-chave virtual reality encontra mais de dois mil artigos.

Na terapia cognitivo-comportamental, o uso de técnicas de exposição é uma intervenção já estabelecida, que pode ser estendida a ambientes virtuais, de acordo com as necessidades específicas do paciente. As exposições em ambientes virtuais têm se provado eficazes no tratamento de vários transtornos ${ }^{2}$ e de vários tipos de pacientes: tanto os que não utilizam computadores quanto os que têm grande contato com esta tecnologia ${ }^{3}$.

Cabe ressaltar, porém, que a introdução tecnológica não implica nova abordagem teórica da psicoterapia. O objetivo é justamente potencializar os tratamentos já existentes e expandir a utilidade das técnicas já utilizadas ${ }^{4}$. Dessa forma, preserva-se a necessidade de considerar os elementos fundamentais da psicoterapia, como a relação terapêutica e o uso pertinente das técnicas já estabelecidas. Castelnuovo et al. ${ }^{5}$ acrescentam que o foco não deve ser a tecnologia, mas o processo psicoterapêutico, que pode ser realçado com o uso de ferramentas tecnológicas. Assim, o uso da realidade virtual, como outras estratégias disponíveis para o campo da psicologia, não deve ser indiscriminado, mas sim coerente com a necessidade do paciente. Por isso, se faz notar a importância de estudos nesta área que apontem as indicações de uso desta ferramenta.

O objetivo deste estudo é apresentar revisão sistemática da literatura sobre realidade virtual e psicoterapia, dando ênfase à técnica de exposição, importante componente da terapia cognitivo-comportamental, e aos estudos que enfocam o tratamento do transtorno de pânico (TP).

\section{MÉTODO}

A busca bibliográfica foi realizada na base de dados PubMed, por meio das palavras-chave: virtual reality e panic disorder. Não houve na busca delimitação do período de publicação dos artigos. A seleção utilizou artigos em língua inglesa. As referências dos trabalhos selecionados foram utilizadas como fonte de consulta. A partir das referências, foram selecionados os artigos sobre TP e realidade virtual e aqueles que continham conceitos sobre exposição a partir de realidade virtual e sobre o conceito de presença. Foram selecionados 28 artigos: 14 originais, 10 revisões de literatura, 2 casos clínicos e 2 comentários sobre artigo publicado.

\section{A realidade virtual e a experiência de presença}

A realidade virtual pode ser definida como uma composição dinâmica e reativa com o ambiente virtual criado por computador e usado para diferentes modalidades de interação humana ${ }^{6}$. Baños ${ }^{7}$ define a realidade virtual como uma experiência humana, uma avançada comunicação de interface que permite ao usuário experimentar "outras realidades". Esta comunicação de interface diz respeito à interação dos componentes computacionais físicos e de informação com os canais sensório-motores do indivíduo (Biocca; Delaney, 1995 apud Riva; Molinari; Vincelli). Steuer ${ }^{8}$ a define como um ambiente real ou simulado em que o indivíduo sente a telepresença (telepresence), ou seja, a experiência de presença a partir de um meio de comunicação.

O ponto essencial destas definições é atribuir ao indivíduo atividade, ou seja, a possibilidade de interação e modificação do ambiente virtual em que está inserido sensorialmente. O indivíduo não fica passivo às imagens e a outros elementos sensoriais disponíveis, não fica restrito à simples observação, ele modifica o ambiente e é passível de modificações e incorporação de informação a partir da interação.

Segundo Riva, Molinari e Vincelli ${ }^{4}$, o grande potencial oferecido pela realidade virtual à psicologia clínica deriva do importante papel que a memória e a imaginação desempenham na psicoterapia. Imagens mentais vêm sendo utilizadas pela psicologia em processos terapêuticos há longa data. Nos transtornos ansiosos, sabe-se que imagens mentais ansiogênicas são responsáveis pela manutenção do medo, da preocupação ou do desconforto, desencadeando pensamentos distorcidos, comportamentos desadaptativos e emoções negativas. Além disso, são capazes de desencadear reações fisiológicas condizentes com aquelas proporcionadas por estímulos ambientais ansiogênicos ${ }^{4}$.

Em adendo, nota-se a dificuldade de distinção entre experiências perceptivas e de imaginação, não apenas em aspectos neurofisiológicos (a localização cerebral de ambos os processos é comum), mas também experimentais e qualitativos (similaridade da vivência de situações reais com experiências de imaginação, dificuldade em distinguir vivências reais de cenas criadas pela imaginação) ${ }^{4}$.

Usar a realidade virtual como um avançado sistema de imagens é uma experiência que pode reduzir a lacuna exis- 
tente entre a imaginação e o mundo real, podendo melhorar a eficácia psicoterapêutica justamente por meio desta aproximação. As imagens mentais podem ser muito úteis na prática clínica: a modificação, a habituação e a substituição de imagens não adaptativas são formas eficazes de tratamento psicológico. O uso da realidade virtual como "avançado sistema de imagens" também é capaz de propiciar ilusão perceptual de não mediação, ou seja, o indivíduo deixa de perceber a existência de mídia em seu ambiente de comunicação e passa a responder como se ela não existisse. A diferença entre ambiente virtual e realidade desaparece ${ }^{4}$.

Esta ilusão de não mediação é parte integrante de um aspecto essencial no estudo da realidade virtual como ferramenta psicoterapêutica, que diz respeito ao conceito de presença (presence) e, principalmente, à sua aplicação prática. De maneira sucinta, presença é sentir-se mais parte do ambiente virtual do que do ambiente real em que se está fisicamente presente. A presença é potencializada quando o usuário está mais envolvido em atividades ou tarefas ${ }^{9}$. Depende do grau de interação e interatividade existente, tanto no ambiente real quanto no virtual ${ }^{10}$.

Dois fatores são importantes para a experiência de presença: envolvimento e imersão ${ }^{11}$. Envolvimento seria o estado psicológico experimentado como conseqüência do foco de energia e atenção em um coerente conjunto de estímulos ou atividades e eventos significativamente relacionados. Já a imersão trata do estado psicológico caracterizado por perceber-se envolvido, incluso, e interagindo com um ambiente que propicie contínuo fluxo de estímulos e experiências.

A presença em um ambiente virtual tem relação com a reprodução de características físicas da realidade. Estímulos auditivos e táteis podem ser acrescentados para aumentar a imersão no ambiente ${ }^{12}$. Porém, sentir o espaço virtual e estar "realmente" imerso nele dependerá mais da forma de locomoção e ação do que outros estímulos sensoriais e a qualidade de imagem ${ }^{10}$.

A estes elementos ainda precisa-se acrescentar a necessidade de criar e compartilhar a rede cultural que dá significado e visibilidade à população e aos objetos que habitam o ambiente virtual. Riva, Molinari e Vincelli ${ }^{4}$ propõem que o conceito de presença inclua quatro pontos fundamentais: reconheça a característica de mediação de qualquer experiência de presença, sempre conceba experiência como imersa em um contexto social, reproduza a ambigüidade inerente às situações cotidianas e enfatize o papel cultural.

Um alto grau de presença está relacionado com maior resposta à terapia, melhores resultados do tratamento e efeitos positivos prolongados ${ }^{3}$. Porém, nem sempre os pacientes ao serem expostos aos ambientes virtuais sentem-se imediatamente imersos, presentes. Alguns pacientes precisam de algumas sessões (duas ou três) para alcançar este resultado, enquanto outros respondem sentindo-se imersos no ambiente virtual logo em seu contato inicial ${ }^{3}$.
Outro conceito distinto de presença, porém complementar, que é um importante elemento experimental para composição de ambientes virtuais, é o julgamento de realidade. Baños et al..$^{13}$ em um artigo que destaca a importância do julgamento de realidade nos ambientes virtuais, e o diferencia do conceito de presença, questionam de que maneira as pessoas decidem o que é real. A resposta para este questionamento pode gerar importantes direcionamentos para a constituição de ambientes virtuais. Assim, os autores usam a argumentação de Brickman (citado por Baños et al.13) para esclarecer o conceito de julgamento de realidade, afirmando que uma experiência é considerada real quando possui correspondência interna e externa. A correspondência interna refere-se à correspondência de comportamentos com sentimentos. Estaria relacionada ao envolvimento, conforme definição de Witmer e Singer ${ }^{11}$, que a presença proporciona. A externa ocorre quando um comportamento tem correspondência com suas conseqüências, estando mais atrelada à imersão.

\section{Tratamentos tradicionais de exposição: exposição ao vivo e imaginária}

A terapia cognitivo-comportamental é a modalidade psicoterapêutica mais estudada no TP e tem demonstrado, nos mais diferentes estudos experimentais, ser bastante eficaz ${ }^{14-16}$. Uma técnica comportamental de extrema importância, principalmente para tratamento da esquiva agorafóbica, é a exposição.

Exposições são procedimentos terapêuticos em que se expõe o cliente aos estímulos ansiogênicos (internos ou externos), a fim de provocar habituação e extinção de respostas ansiosas e agorafóbicas. Há diferentes modalidades de exposição, por exemplo, ao vivo e imaginária. Tanto a exposição ao vivo quanto a imaginária visam ao fortalecimento da autoeficácia dos pacientes. A exposição ao vivo utiliza-se de exposições hierárquicas (graduais) às situações temidas ou evitadas, de modo prolongado, visando a queda da ansiedade diante do estímulo ansiogênico. As exposições imaginárias utilizam-se da construção de imagens mentais que contenham os estímulos que são ansiogênicos para os pacientes.

\section{Exposições virtuais}

Riva $^{17}$ descreve a realidade virtual como um meio tão efetivo quanto a realidade em induzir respostas emocionais. Isso porque os softwares de realidade virtual podem ser constituídos de maneira que os ambientes virtuais reproduzam situações ansiogênicas, muito próximas à realidade, que sirvam como cenário para exposições no tratamento de diferentes transtornos psiquiátricos. São capazes de gerar estímulos de maior magnitude do que outras técnicas já estabelecidas ${ }^{18}$. Da mesma forma que ocorre nas exposições ao vivo e imaginária, é possível construir com o paciente uma lista com a hierarquia das situações e dos estímulos temidos corres- 
pondentes à realidade. Os estímulos temidos são integrados em ambientes virtuais que permitem interação, possibilitando ao paciente que aja em tais cenários da mesma maneira que agiria em um ambiente real ${ }^{19}$. O sentimento de presença que os ambientes virtuais permitem experimentar e o envolvimento sensório-motor (proporcionado por diferentes estímulos sensoriais e por traçadores de posição) proporcionam sensação de imersão realista e vívida, maior do que o sujeito poderia sentir na construção de cenários por sua própria imaginação ${ }^{20}$. A implementação da realidade virtual nos exercícios de exposição pode ser indicada como alternativa, tanto para as exposições imaginárias quanto para as ao vivo, podendo ser também um passo intermediário para o confronto direto com as situações temidas.

O uso de ambientes virtuais destinados ao tratamento de exposição é bastante eficaz quando comparado aos resultados com exposição imaginária ${ }^{3,19}$. Sabe-se que para que a habituação ocorra de maneira mais eficaz, é necessário que o paciente sinta ativação, tanto de maneira objetiva (ansiedade medida por instrumentos objetivos, como medidas fisiológicas) quanto subjetiva (auto-relato de ansiedade). É o que diz o conceito de sinchrony, que ocorre quando as medidas objetivas e subjetivas acontecem juntas durante o tratamento, sendo mais propício advir em situações de grande ativação ansiogênica (Rachman; Rodgson, 1974 citado por Wiederhold; Wiederhold ${ }^{21}$ ). Na exposição imaginária talvez não ocorra imersão nos cenários imaginários passível de ativação objetiva e subjetiva, já que os pacientes podem mais facilmente evitar cognitivamente a exposição aos cenários, diminuindo a ansiedade experimentada e dificultando a habituação ${ }^{3}$. Além disso, as exposições por realidade virtual proporcionam maiores mudanças no senso de autoeficácia do que a exposição imaginária, já que os ambientes virtuais aumentam a possibilidade de o paciente manejar com sucesso situações muito ansiogênicas (como será visto em relação à exposição ao vivo(22.

Nos cenários virtuais, diferentemente dos cenários imaginários, as cenas para exposição são constituídas por estímulos concretos que facilitam o envolvimento emocional, aumentando a probabilidade de extinção da resposta de medo ${ }^{19}$. Esta possibilidade é uma alternativa para os pacientes que denotam dificuldade em imaginar cenas evocativas de ansiedade ou manter-se imaginando tais cenas ${ }^{21}$.

Além disso, a exposição em ambientes de realidade virtual admite o preenchimento de uma lacuna: permite que o paciente se exponha aos estímulos ansiogênicos de maneira vívida, como se fossem reais, quando a exposição ao vivo não é possível ou a ansiedade diante da situação real ainda é extrema, dificultando a entrada ou a permanência do paciente à situação de exposição, diminuindo também o abandono ao tratamento ${ }^{23,24}$.

Uma característica importante é que as exposições podem ser administradas em consultório, sendo mais conve- nientes (maior facilidade em criar situações adequadas do que procurá-las na vida real, além da possibilidade de exposição às situações de escassa ocorrência) e controladas (a intensidade dos estímulos provocadores de ansiedade é escolhida por paciente e terapeuta, respeitando a ordem hierárquica preestabelecida, diminuindo a possibilidade de imprevistos); garantindo a segurança inicial para exposições a estímulos muito ansiogênicos e preservando a confidencialidade do tratamento. Além de proporcionar a segurança inicial para exposições ao vivo, conforme citado anteriormente, a realidade virtual também pode ser utilizada em outros momentos do tratamento, como em situações de crise, ajudando o paciente a superar tal momento e prosseguir no tratamento ${ }^{17}$.

Preocupações focais dos pacientes também podem ser isoladas e abordadas de modo mais efetivo nos ambientes virtuais ${ }^{6,19}$. O cenário virtual, pela segurança que proporciona, pode também facilitar a expressão de pensamentos e sentimentos que em situações reais poderiam ser difíceis de serem abordados, fortalecendo também a relação terapeuta-paciente ${ }^{17}$.

Uma característica importante dos softwares de realidade virtual é a flexibilidade de constituição dos ambientes virtuais. Alguns softwares já permitem manipular grande quantidade de estímulos, estruturando o ambiente conforme as necessidades dos pacientes, além de monitorar as respostas do paciente na interação com o cenário virtual. Estas possibilidades incrementam a efetividade terapêutica do tratamento de exposição virtual20.

As respostas individuais aos ambientes virtuais são variadas, assim como as necessidades específicas de cada paciente ${ }^{3}$. Daí a importância da referida flexibilidade dos ambientes virtuais, que permitam modificações pertinentes para atender à singularidade dos casos abordados. Além disso, a habilidade e o conhecimento do psicoterapeuta também são postos em foco, já que este também precisa ter recursos adequados para a avaliação das necessidades específicas dos pacientes, para definir como tais necessidades podem ser inseridas na reconstrução dos ambientes virtuais e, também, para decidir o conteúdo das intervenções necessárias no momento da exposição. Neste último aspecto, Wiederhold e Wiederhold ${ }^{3}$ coletaram dados interessantes sobre as diferentes formas de intervenção do psicoterapeuta requeridos pelos pacientes. Concluíram que dependendo do tipo de fobia apresentado, o paciente exposto ao ambiente virtual requisitava a participação do psicoterapeuta de maneira diferente. Pacientes com medo de voar preferiram não ser interrompidos pelo terapeuta quando questionados sobre seu nível de ansiedade; já os pacientes com ansiedade social sentiram-se mais capazes de verbalização durante a exposição e também se perceberam mais como fazendo parte do cenário virtual. 
A aliança terapêutica é um componente fundamental do trabalho psicoterapêutico com realidade virtual, assim como em qualquer modalidade de psicoterapia, porém, segundo Cárdenas et al. ${ }^{24}$, alguns autores ainda citam este aspecto como um ponto fraco da psicoterapia de exposição por realidade virtual. Em contrapartida, os próprios autores ${ }^{24}$ apontam estudos que demonstram o contrário, como este pode ser um elemento diferencial. Um deles demonstra que há equivalência na satisfação com a psicoterapia tradicional e a psicoterapia complementada por novas tecnologias, além de maior aceitação da terapia de realidade virtual quando comparada à terapia individual.

\section{Medidas fisiológicas durante a exposição virtual}

Na psicoterapia com uso de realidade virtual é importante que os pacientes experimentem alterações fisiológicas e ansiedade fóbica similares àquelas que sentem em situações da vida real23. Até mesmo indivíduos não-fóbicos apresentam alguma ativação fisiológica quando expostos pela primeira vez a um ambiente virtual, mas estabilizam sua fisiologia em período médio de 20 minutos $^{3}$.

Pacientes fóbicos apresentam respostas significativamente diferentes de sujeitos não-fóbicos na exposição ao ambiente virtual, porém tal diferença tende a diminuir com a habituação ${ }^{25}$. A fisiologia é uma forma de avaliar os resultados do tratamento a partir de realidade virtual.

São poucos os estudos que mensuram respostas fisiológicas. Wiederhold et al. ${ }^{26}$ verificaram em um estudo que participantes e pacientes acompanhados por dois anos não mostraram mudanças na temperatura periférica da pele. $\mathrm{O}$ ritmo cardíaco também não apresentou variações significativas, exceto nos pacientes que tiveram ataques de pânico durante a exposição. Também não foi sensível a mudança no ritmo respiratório. A única medida que mostrou alterações significativas foi a condutância da pele. As respostas fisiológicas percebidas foram as mais rápidas a partir da apresentação do estímulo visual e também durante o tratamento, conforme ocorria o processo de dessensibilização.

Há também na literatura pesquisas com medidas eletroencefalográficas que, de modo similar às outras medidas fisiológicas, estão em fase inicial de investigação, ainda não apontando relações conclusivas que ajudem no aprimoramento do tratamento ${ }^{27,28}$.

\section{Tratamento do TP a partir de realidade virtual}

Jang et al. ${ }^{29}$ submeteram sujeitos diagnosticados com agorafobia ao tratamento de exposição virtual, que incluía treino em relaxamento, em dez diferentes cenários, para verificar sua eficácia. Imediatamente ao participar das sessões de realidade virtual, os sujeitos relataram alterações físicas, como suor e palpitações. Ao decorrer da exposição não foram relatadas mudanças fisiológicas e também não houve aumento nos escores da escala Subjective Units of Distress Scale (SUDS). Verificou-se que a maioria dos participantes não conseguiu sentir-se imerso nos ambientes virtuais, e as exposições foram suspensas após a segunda sessão. Os autores ${ }^{29}$ apontaram alguns fatores como possíveis variáveis que influenciaram de maneira negativa a imersão dos sujeitos nos cenários virtuais, como o tempo (20 minutos) de uso do capacete com visor (head mounted display) que deixou os participantes desconfortáveis e seu campo de visão de 50 graus que dificultou a imersão; a presença contínua do terapeuta próximo ao sujeito durante toda a exposição; a luz que permitia visão pela fenda do capacete com visor; o desconforto dos sensores acoplados ao corpo para mensurar dados fisiológicos; e a indefinição sobre os limites das verbalizações do terapeuta.

Em um estudo que comparava três grupos-controle, TCC com exposição virtual e apenas TCC -, Vincelli et al. ${ }^{20}$ verificaram que nos dois grupos de tratamento houve redução significativa de ataques de pânico, nível de depressão, estado e traço de ansiedade. A diferença foi constatada no tempo de obtenção destes resultados: o grupo de tratamento com exposições virtuais chegou a tais resultados com apenas oito sessões, enquanto o outro grupo de tratamento foi submetido a 12 sessões.

Choi et al. ${ }^{6}$ testaram a eficácia de um tratamento para TP com agorafobia de exposição virtual de quatro sessões comparado a um tratamento cognitivo-comportamental (panic control program) de 12 sessões. Ambos os grupos melhoram significativamente após o tratamento, porém a efetividade em longo prazo foi maior no grupo que não obteve exposições virtuais no protocolo de tratamento. Outro achado importante foi que a terapia cognitivo-comportamental foi mais efetiva na modificação das medidas relacionadas à avaliação cognitiva da ansiedade, enquanto o tratamento com exposições virtuais se sobressaiu no manejo da ansiedadetraço.

\section{DISCUSSÃO}

As potencialidades que a realidade virtual acrescenta ao tratamento de exposições são significativas e uma grande vantagem é a aceitabilidade deste tipo de tratamento por parte dos pacientes. Garcia-Palacios et al.30 mostram que 80\% dos participantes de seu estudo preferiam exposições virtuais a exposições ao vivo. É possível que este resultado reflita algum tipo de esquiva aos estímulos reais, porém é vantajoso que o paciente considere inicialmente a exposição virtual como forma de tratamento, o que pode funcionar como preparação para futuras exposições ao vivo.

Porém, apesar de as vantagens do uso de realidade virtual, pode-se destacar inúmeras limitações da utilização desta tecnologia. Alguns pacientes relataram não gostar dos estímulos 
visuais da realidade virtual ou relataram sentir sonolência durante a exposição virtual ${ }^{3}$. Há pacientes que sentiram enjôo, 0 que pode influenciar no abandono do tratamento ${ }^{19}$.

Existem também as limitações impostas pelo software, que muitas vezes restringe-se ao protocolo criado. Requisições não incluídas no programa não podem ser facilmente providenciadas, dificultando a adequação dos ambientes virtuais às necessidades específicas de cada paciente.

A falta de protocolos padronizados também ainda é uma deficiência dessa forma de tratamento, que aponta a necessidade de pesquisas para sua constituição. A publicação dos protocolos é de vital importância para diminuir custos e tempo, compartilhando pontos fracos e fortes e evitando a elaboração de tratamentos por tentativas e erros ${ }^{17}$.

Segundo Jang et al. ${ }^{23}$, a proximidade do terapeuta com o paciente pode inibir a imersão, já que, principalmente em momentos de maior ansiedade, os pacientes asseguraramse da presença do terapeuta, o que pode dificultar o processo de habituação.

Riva e Wiederhold ${ }^{22}$ apontam a carência de estudos controlados no campo da realidade virtual que demonstrem suas vantagens clínicas e econômicas, apesar de a teoria que respalda seu uso esteja consolidada. No Brasil, este tipo de tecnologia está longe do fácil acesso por causa dos custos dos equipamentos necessários e da construção dos ambientes virtuais.

\section{CONCLUSÃO}

Verificam-se os bons resultados da realidade virtual aplicada ao tratamento do TP. Entretanto, apesar de grande avanço, muito do potencial da realidade virtual está ainda inexplorado. É importante que novos ambientes virtuais sejam criados para que investigações controladas a respeito de sua aplicação clínica sejam realizadas, atentando para a divulgação desta prática que contribui com o aprimoramento dos ambientes e a velocidade com que ocorre, evitando que diversos pesquisadores tenham de cometer semelhantes tentativas e erros no desenvolvimento de seu trabalho. Neste processo de criação e investigação, é fundamental que as experiências clínicas a partir dos ambientes virtuais estejam relacionadas às experiências reais, em um contexto flexível que conjugue aspectos culturais, físicos e cognitivos pertinentes.

\section{REFERÊNCIAS}

1. Wiederhold BK. Virtual reality in the 1990s: what did we learn? Cyberpsychol Behav. 2000;3(3):311-4.

2. Rothbaum BO, Hodges L, Alarcon R, Ready D, Shahar F, Graap K, et al. Virtual reality exposure therapy for PTSD Vietnam veterans: a case study. J Trauma Stress. 1999;12:263-71.

3. Wiederhold BK, Wiederhold MD. Lessons learned from 600 virtual reality sessions. Cyberpsychol Behav. 2000;3(3):393-400.
4. Riva G, Molinari E, Vincelli F. Interaction and presence in the clinical relationship: virtual reality (vr) as communicative medium between patient and therapist. IEEE Trans Inf Technol Biomed. 2002;6(3):198-205.

5. Castelnuovo G, Gaggioli A, Mantovani F, Riva G. From psychotherapy to e-therapy: the integration of traditional techniques and new communication tools in clinical settings. Cyberpsychol Behav. 2003;6(4):375-82.

6. Choi Y, Vincelli F, Riva G, Wiederhold BK, Lee J, Park K. Effects of group experiential cognitive therapy for the treatment of panic disorder with agoraphobia. Cyberpsychol Behav. 2005;8(4):387-93.

7. Baños RM. Commentary on Riva, G., virtual reality in psychotherapy: review. Cyberpsychol Behav. 2005;8(3):232-3.

8. Steuer J. Defining virtual reality: dimensions determining telepresence. Journal of Communication. 1992;42(4):73-93.

9. Coelho CM, Santos JA, Silvério J, Silva CF. Virtual reality and acrophobia: one-year followup and case study. Cyberpsychol Behav. 2006;9(3):336-41.

10. Sastry L, Boyd DRS. Virtual environments for engineering applications. Virtual Reality: Res., Development, Applicat. 1998;3(4):235-44.

11. Witmer BG, Singer MJ. Measuring presence in virtual environments: a presence questionnaire. Presence: Teleoperators and Virtual Environments. 1998;7(3):225-40.

12. Emmelkamp PMG. Commentary on Riva G, Virtual Reality in Psychotherapy: Review. Cyberpsychol Behav. 8(3): 235-7, 2005.

13. Baños RM, Botella C, Garcia-Palacios A, Villa H, Perpina C, Alcañiz M. Presence and reality judgment in virtual environments: a unitary construct? Cyberpsychol Behav. 2000;3(3):327-35.

14. Haby MM, Donnelly M, Corry J, Vos T. Cognitive behavioural therapy for depression, panic disorder and generalized anxiety disorder: a meta-regression of factors that may predict outcome. Aust N Z J Psychiatry. 2006;40:9-19.

15. Gould RA, Otto MW, Pollack MH. A meta-analysis of treatment outcome for panic disorder. Clin Psychol Rev 1995;15(8):819-44.

16. Clum GA, Clum GA, Surls R. A meta-analysis for panic disorder. J Consult Clin Psychol. 1993;61:317-26.

17. Riva G. Virtual reality in psychotherapy: review. Cyberpsychol Behav. 2005;8(3):220-30.

18. Andrews T. Commentary on Riva, G., virtual reality in psychotherapy: review. Cyberpsychol Behav. 2005;8(3):231-2.

19. Maltby N, Kirsch I, Mayers M, Allen GJ. Virtual reality exposure therapy for the treatment of fear of flying: a controlled investigation. J Consult Clin Psychol. 2002;70(5):1112-8.

20. Vincelli F, Anolli L, Bouchard S, Wiederhold BK, Zurloni V, Riva G. Experiential cognitive therapy in the treatment of panic disorders with agoraphobia: a controlled study. Cyberpsychol Behav. 2003;6(3):321-8.

21. Wiederhold BK, Wiederhold MD. Three-year follow-up for virtual reality exposure for fear of flying. Cyberpsychol Behav. 2003;6(4):441-5.

22. Riva G, Wiederhold BK. Guest editorial: introduction to the special issue on virtual reality environments in behavioral sciences. IEEE Trans Inf Technol Biomed. 2002;6(3):193-7.

23. Jang DP, Ku JH, Choi YH, Wiederhold BK, Nam SW, Kim IY, et al. The Development of virtual reality therapy (vrt) system for the treatment of acrophobia and therapeutic case. IEEE Trans Inf Technol Biomed. 2002;6(3):213-7.

24. Cárdenas G, Muñoz S, González M, Uribarren G. Virtual reality applications to agoraphobia: a protocol. Cyberpsychol Behav. 2006;9(2):248-50.

25. Wiederhold BK, Jang DP, Kim SI, Wiederhold MD. Physiological monitoring as an objective tool in virtual reality therapy. Cyberpsychol Behav. 2002;5(1):77-82.

26. Wiederhold BK, Jang DP, Kim SI, Wiederhold MD. Physiological Monitoring as an Objective Tool in Virtual Reality Therapy. Cyberpsychol Behav. 5(1): 77-82, 2002.

27. Othmer S, Kaiser D. Implementation of virtual reality in EEG biofeedback. Cyberpsychol Behav. 2000;3(3):415-20.

28. Baumgartner T, Valko L, Esslen M, Jancke L. Neural correlate of spatial presence in an arousing and noninteractive virtual reality: an eeg and psychophysiology study. Cyberpsychol Behav. 2006;9(1):30-45.

29. Jang DP, Ku JH, Shin MB, Choi YH, Kim SI. Objective validation of the effectiveness of virtual reality psychotherapy. Cyberpsychol Behav. 2000;3(3):369-74.

30. Garcia-Palacios A, Hoffman HG, See SK, Tsai A, Botella C. Redefining therapeutic success with virtual reality exposure therapy. Cyberpsychol Behav. 2001;4:341-8. 